\title{
Multiple TAS-OSTBC in MIMO with Outer BCH Concatenation under Nakagami-m Fading
}

\author{
Vaibhav Hendre, Nitin Deotale
}

\begin{abstract}
Transmit Antenna Selection (TAS) technique with Orthogonal Space Time Block Code (OSTBC) addresses the problem of hardware complexity in multiple antenna systems and improves Bit Error Rate (BER) significantly by using various antenna selection algorithms. TAS technique is not able to provide the coding gain. To achieve the coding gain benefits with improvement in BER, it is necessary to concatenate TAS system with an outer code. In literature, it is observed that the concatenation of Forward Error Correction (FEC) codes and Space Time Block Code (STBC) for MIMO systems provides the high coding gain with improved BER. In this paper, a multiple TAS-OSTBC system is proposed with concatenation of channel coding techniques such as BCH and TURBO. The proposed system is concatenated with $\mathrm{BCH}$ as outer code in series with TURBO as inner code. This combination provides the capacity benefits of TAS-OSTBC system with improved coding gain. The channel is assumed to be faded by Nakagami- $m$ fading. It is observed that for multiple TAS-OSTBC system this new concatenation of BCH-TURBO code gives improvement of $5 \mathrm{~dB}$ at 10-6 as compared to concatenation of TURBO code at $m=0.5$ for Nakagami- $m$ fading parameter.
\end{abstract}

Keywords : Bit error Rate (BER), Bose Chaudhuri Hocquenghem (BCH) code, Nakagami- m Fading, TURBO Codes, Orthogonal Space Time Block Codes (OSTBC), Transmit Antenna Selection (TAS)

\section{INTRODUCTION}

Multiple Input Multiple Output (MIMO) systems are used in all recent wireless communication system as it fulfill the needs of higher data rates with efficient and reliable digital transmission. MIMO systems can be implemented either to provide high data rates by using spatial multiplexing or to provide the diversity benefits by using Space Time Coding (STC). The hardware complexity problem of MIMO system is addressed by a technique called as Antenna Selection (AS) proposed in [1] in which the subset of antennas which are less than the number of transmitting and or receiving antennas selected based on the channel state information of the system.

Revised Manuscript Received on August 30, 2019.

* Correspondence Author

Vaibhav Hendre*, Department of Electronics \& Telecommunication Engineering, G. H. Raisoni College of Engineering \& Management, Pune, Maharashtra, India

Nitin Deotale, Department of Electronics \&amp; Telecommunication Engineering, Lokmanya Tilak College of Engineering, Navi Mumbai, Maharashtra, India.

(C) The Authors. Published by Blue Eyes Intelligence Engineering and Sciences Publication (BEIESP). This is an open access article under the CC BY-NC-ND license (http://creativecommons.org/licenses/by-nc-nd/4.0/)
Transmit Antenna Selection (TAS) is more popular as it selects the best possible subset of antenna for transmission using the channel state information feedback by the receiver [2]. TAS-MIMO system is presented in [1] by considering Maximal Ratio Combining (MRC) technique where single subset of antenna is selected for transmission purpose. The Bit Error Rate (BER) performance of TAS system was carried out for various fading channels in [3]-[5]. TAS systems can reduce the BER considerably based on the different antenna selection algorithms. TAS technique is not able to provide the coding gain. To achieve the coding gain benefits with improvement in BER, it is necessary to concatenate TAS system with an outer code. In literature, it is observed that the concatenation of Forward Error Correction (FEC) codes and Space Time Block Code (STBC) for MIMO systems provides the high coding gain with improved BER. The concatenation of STBC with different FEC codes which includes Convolutional codes (CC), turbo codes and Trellis Coded Modulation (TCM) were presented in [6]. The performance of MIMO system concatenated with LDPC code is proposed in [7]. The concatenation of STBC with turbo codes was proposed in [8]. It was observed in [9] that Signal to Noise Ratio (SNR) gain of more than $3 \mathrm{~dB}$ is achieved for (2 X 2) MIMO systems with TAS concatenated with Turbo codes. The MIMO-OSTBC system is proposed in [10] by considering $\mathrm{BCH}$ code under Rayleigh fading and the performance of BCH code for SC-FDM-IDMA Scheme is presented in [11].

In this paper, the TAS-OSTBC system is initially concatenated with various FEC codes to verify the performance of concatenation combinations. The BCH codes when concatenated with Turbo codes provide significant improvement in coding gain as presented in [12]. Therefore, this paper further proposes a multiple TAS-OSTBC concatenated with outer $\mathrm{BCH}$ code in series with inner TURBO code. This combination provides the capacity benefits of TAS-OSTBC system with improved coding gain. The fading channel is considered here as Nakagami- $\mathrm{m}$ fading channel which includes both Rician and Reyleigh fading and a special case of practical fading with $\mathrm{m}=0.5$.

\section{PROPOSED TAS-OSTBC SYSTEM CONCATENATED WITH OUTER BCH}

The proposed MIMO-OSTBC system implementing TAS technique and concatenated with outer BCH code is as shown in Fig. 1. 


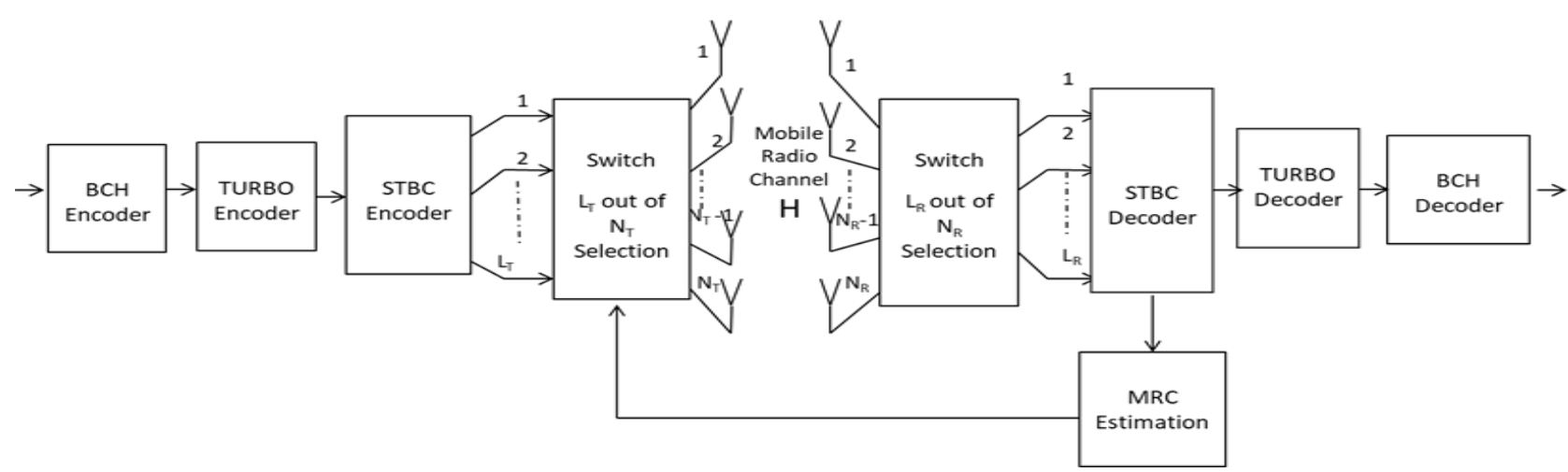

Fig. 1 TAS-OSTBC system with concatenated with outer BCH Code

The numbers of transmitting antennas are referred as $\mathrm{N}_{\mathrm{T}}$ and number of receiving antennas as $\mathrm{N}_{\mathrm{R}}$. The MIMO system is refereed as $\left(\mathrm{N}_{\mathrm{T}}, \mathrm{N}_{\mathrm{R}}\right)$ system without antenna selection. The switch selects $\mathrm{L}_{\mathrm{T}}$ out of $\mathrm{N}_{\mathrm{T}}$ antennas for transmission based on the estimated CSI received from receiver. If we consider TAS system selecting $\mathrm{L}_{\mathrm{R}}$ antennas, it is referred as $\left(\mathrm{N}_{\mathrm{T}^{\prime}} \mathrm{L}_{\mathrm{R}}, \mathrm{N}_{\mathrm{R}}\right)$ system. The channel matrix is denoted by $\mathrm{H}$ and has size of $\left(\mathrm{L}_{\mathrm{T}} \mathrm{X} \mathrm{L}_{\mathrm{R}}\right)$. The channel coefficients are denoted by $\mathrm{h}_{\mathrm{i} j \mathrm{j}}$, where, $1 \leq \mathrm{i} \leq \mathrm{L}_{\mathrm{T}}$ and $1 \leq \mathrm{j} \leq \mathrm{L}_{\mathrm{R}}$.

The channel coefficients are considered as the samples of Nakagami- $m$ fading function. Here, the channel gain $\left|h_{i, j}\right|$ follows a Nakagami- $m$ fading distribution given by PDF

$$
\mathrm{p}_{\mathrm{Y}}(\mathrm{\gamma})=\frac{\gamma^{\mathrm{mL}_{\mathrm{R}}-1}}{\left(\frac{\bar{\gamma}}{\mathrm{m}}\right)^{\mathrm{mL}_{\mathrm{R}}} \Gamma\left(\mathrm{mL}_{\mathrm{R}}\right)} \exp \left(-\mathrm{m} \frac{\gamma}{\bar{\gamma}}\right)
$$

where, $\gamma$ is the instantaneous SNR estimated at the receiver, the gamma function is represented as $\mathrm{r}(\mathrm{z})=\int_{0}^{\infty} \mathrm{x}^{\mathrm{z}-1} \mathrm{e}^{-\mathrm{x}} \mathrm{dx}$, and $\mathrm{m}$ - is the fading parameter for Nakagami fading channel. If the transmitted signal is $\mathbf{x}$, the channel gain is denoted as $\mathrm{h}$ and additive white noise as $\mathrm{n}$, the received signal $y$ is given as

$$
\mathrm{y}=\mathrm{h} * \mathrm{x}+\mathrm{n}
$$

The pilot based training technique is used to estimate the received SNR. The Maximal Ratio Combining (MRC) technique is used at the receiver to find the index of the subset with higher SNR. This index of the subset of single selected transmit antenna is denoted by ${ }^{t} \mathrm{~T}^{t}$ and is given as [4]

$$
I=\underset{1 \leq i \leq L_{\mathrm{L}}}{\operatorname{argmax}}\left\{\mathrm{C}_{\mathrm{i}}=\sum_{\mathrm{j}=1}^{\mathrm{L}_{\mathrm{T}}}\left|\mathrm{h}_{\mathrm{i}_{\mathrm{i}, \mathrm{j}}}\right|^{2}\right\}
$$

To improve further coding gain it is necessary to concatenate OCTBC system with some outer code as proposed in [13]. Turbo codes are used here due to their remarkable performance.

The multiple antenna selection is considered here based upon suboptimal selection method as proposed in [14]. The first subset of antenna is selected which provides the maximum value of MRC as per equation (3). The indices of antenna subset calculated by MRC are further arranged in descending order. Here $\mathrm{L}_{\mathrm{T}}$ is the number of antennas selected for transmission and $\mathrm{C}(1)$ are the indices of antennas organized in descending order as $\mathrm{C}(1) \leq \mathrm{C}(2) \leq \cdots \leq \mathrm{C}\left(\mathrm{L}_{\mathrm{T}}\right)$. For single TAS/MRC case, the instantaneous SNR $\gamma\left(\mathrm{L}_{\mathrm{T}}\right)$ is given as $\gamma\left(\mathrm{L}_{\mathrm{T}}\right)=\mathrm{C}\left(\mathrm{L}_{\mathrm{T}}\right) \bar{\gamma}$.

The norm based method is used for selecting the subset of antennas. Let $\mathrm{h}_{\mathrm{j}}$ where $\left(\mathrm{j}=1_{w}, \ldots \ldots \mathrm{L}_{\mathrm{T}}\right)$ is the columns of the channel matrix $H$. The columns of the matrix $h_{\mathrm{j}}$ are arranged in the increasing order of Norms, $\left\|h_{\mathrm{i} 1}\right\| \geq \ldots \geq\left\|h_{\mathrm{iL}_{T}}\right\|$. Here, $\mathrm{i}_{\mathrm{k}} \in\left\{1_{s} 2 \ldots \mathrm{L}_{\mathrm{T}}\right\}$ The best possible transmit antenna is selected which minimizes the BER.

The pairwise error probability is denoted by

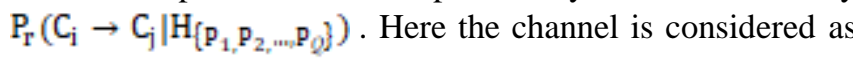

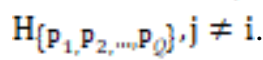

The pair-wise error probability is given as [5]

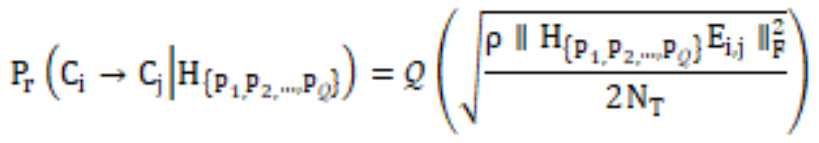

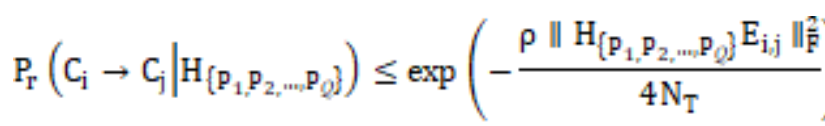

Here, the number of antenna subset selected that minimizes the upper bound as per Eq. (4) is denoted by Q and is given as

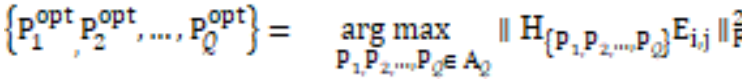

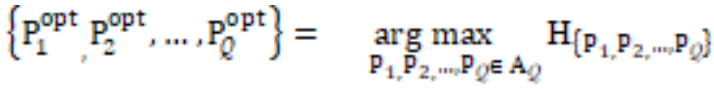

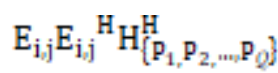

Further, with $Q$ selected antennas of $\left\{\mathrm{P}_{i}\right\}_{\mathrm{i}=1}^{\mathcal{Q}}$, the average $\mathrm{SNR}$ is given as

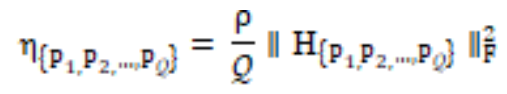


The subset of antenna is selected based upon equation (7) and (8). It is assumed that $Q$ numbers of antennas are selected by using optimal selection process. In this case, the average received SNR is given as

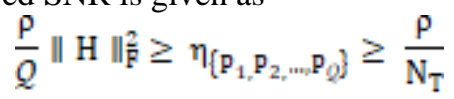

The performance analysis for single TAS-MIMO system is presented in [14] under Nakagami- $\mathrm{m}$ fading channel is extended in this paper for multiple antenna selection case. The instantaneous BER is given as $P_{E}(\beta)=Q(\sqrt{k \beta \gamma})$ where $\beta=\frac{Y}{\gamma}$ which is channel power gain for BPSK. As per [14], the average BER is given as

$$
P_{E}=\frac{2^{t} a \Gamma\left(t+\frac{3}{2}\right)}{\sqrt{\pi}(t+1)}(k \bar{\gamma})^{-[t+1)}+o\left(\bar{\gamma}^{-[t+1)}\right)
$$

The average BER for single TAS-OSTBC system by considering Nakagami- $\mathrm{m}$ fading channel is given as

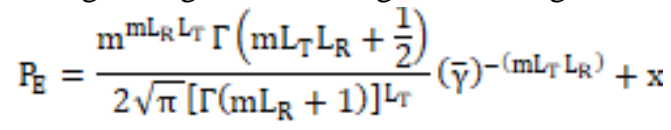

where, $\mathrm{k}=2$ is assumed for BPSK and $\mathrm{x}=\mathrm{o}\left(\bar{\gamma}^{\left.-\mathrm{mL}_{\mathrm{T}} \mathrm{L}_{\mathrm{R}}\right)}\right.$ and $\mathrm{m}$ and $\mathrm{L}_{\mathrm{R}}$ are constant. The case of single antenna selection is represented as, $\left(\mathrm{L}_{\mathrm{T}}, 1_{s} \mathrm{~L}_{\mathrm{R}}\right)$ and the $\mathrm{BER}$ equation is given as

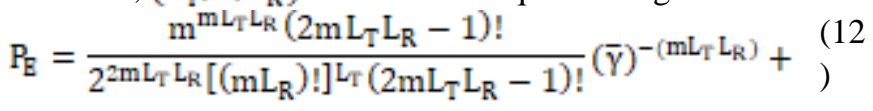

The analysis presented here is extended here for multiple TAS case. For the analysis purpose the case of $(4 \mathrm{x} 4) \mathrm{MIMO}$ with 2 antennas selection is considered. For the combination of $\left(4,1 ; L_{R}\right)$, The probability of error is given as

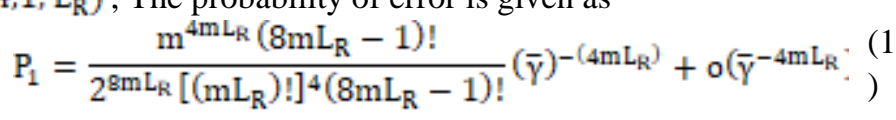

Fig. 3 BER analysis of proposed TAS-OSTBC-BCH-TURBO Code with $\mathrm{m}=0.5$

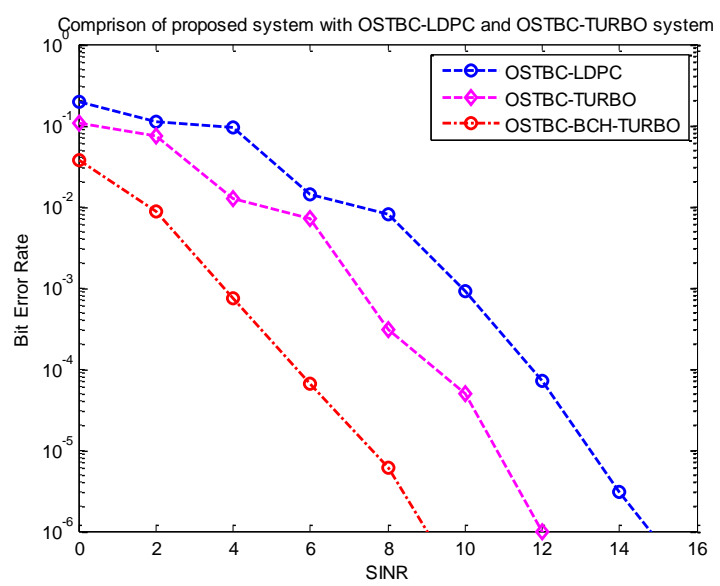

Fig. 4 BER analysis comparison of proposed TAS-OSTBC system with OSTBC-LDPC and OSTBC-TURBO system at $\mathrm{m}=0.5$ code is better as compared to CC, BCH, RS and TCM code. OSTBC-TURBO gives BER which is around $10^{-5}$ for SINR of $10 \mathrm{~dB}$ whereas the same will be obtained above $16 \mathrm{~dB}$ for TAS
without FEC. Therefore for the proposed system, TURBO $10 \mathrm{~dB}$ whereas the same will be obtained above $16 \mathrm{~dB}$ for TAS
without FEC. Therefore for the proposed system, TURBO codes are used as inner code for the concatenation with

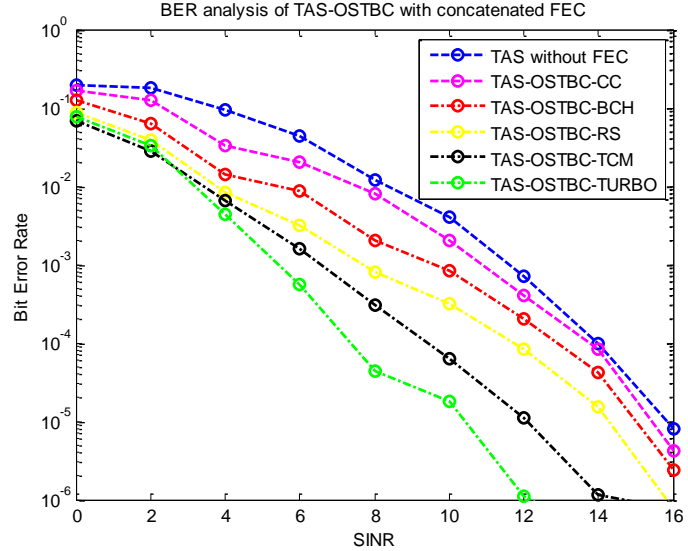

Fig. 2 BER analysis of TAS-OSTBC system with concatenated FEC Code

TAS-OSTBC-BCH-TURBO gives BER in the range of $10^{-5}$ for SINR of $8 \mathrm{~dB}$ whereas the same is achieved for more than $14 \mathrm{~dB}$ for TAS-OSTBC-TURBO case as shown in Fig.3.

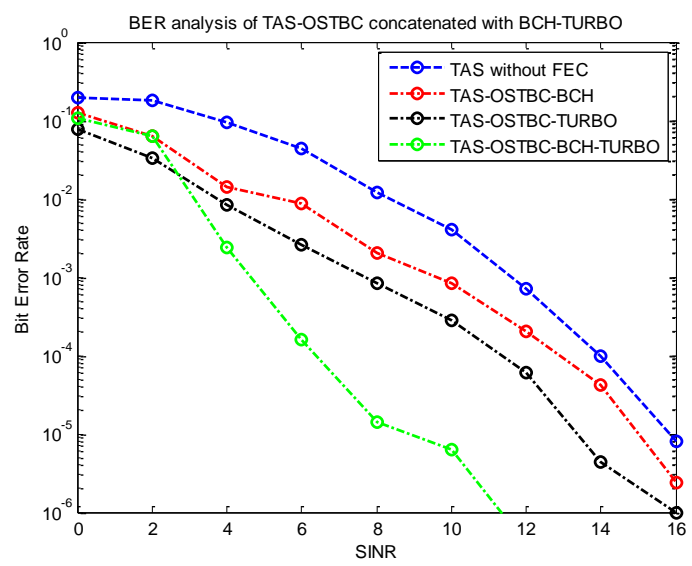

Similarly, the $P_{2}$ is calculated as presented in [15]. The value of $P_{1}$ and $P_{2}$ is calculated from equation (7) for the case of $\left(4,2, L_{R}\right)$. The BER for $\left(4,2 ; L_{R}\right)$ case i.e. selection of 2 subset antennas from 4 transmitting antennas is carried out by using sub optimal method as per equation (7). The equation (8) ensures the minimization of upper bound of error probability and selects the proper subset of antenna.

\section{PERFORMANCE ANALYSIS OF PROPOSED TAS-OSTBC SYSTEM}

The performance analysis of proposed TAS-OSTBC system is verified initially for generalized FEC codes. The channel is assumed to be faded by the Nakagami- $\mathrm{m}$ fading channel coefficients. The performance of TAS-OSTBC system concatenated with generalized FEC codes is verified. Figure 2 shows that, the performance of OSTBC-TURBO Retrieval Number F8409088619/20190BEIESP 
The proposed TAS-OSTBC system concatenated with BCH-TURBO is compared with TAS-OSTBC system concatenated with TURBO code and TAS-OSTBC system concatenated with LDPC code as given in [7]. It is observed from Fig. 4 that BER of 10-4 is achieved at $6 \mathrm{~dB}$ in proposed system as compared to OSTBC-LDPC system which achieved it at $12 \mathrm{~dB}$.

The BER analysis of proposed system is carried out for multiple TAS systems as shown in Fig. 5. Here, the conventional MIMO system without TAS is referred as $(4 \times 4)$ MIMO system. The single TAS system from (4x4) MIMO is referred as $(4: 1,4)$ system. Subsequently, $(4: 2,4)$ and $(4: 3,4)$ refers 2 and 3 antenna selection from 4 transmit antennas respectively. It is observed from the figure that the performance of the system enhances with increase in number of selected subset of transmitting antennas.

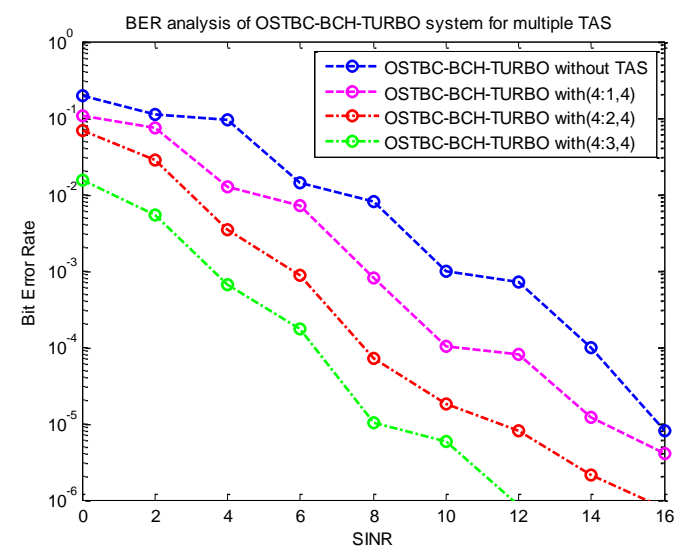

Figure 5 BER analysis of OSTBC-BCH-TURBO system for multiple TAS system with $\mathrm{m}=0.5$

The performance analysis of the proposed system is also verified for different values of $\mathrm{m}$ in Nakagami fading channel. The fading parameter with $\mathrm{m}=1$ is a special case of Rayleigh fading. It is observed from the Fig. 6 that the performance of the system improves by increasing the value of $\mathrm{m}$. The $\mathrm{m}=0.5$ is a special case of Nakagami- $\mathrm{m}$ fading and considers the practical fading scenario for wireless communication. It is observed that the proposed system outperform even for the case of $\mathrm{m}=0.5$.

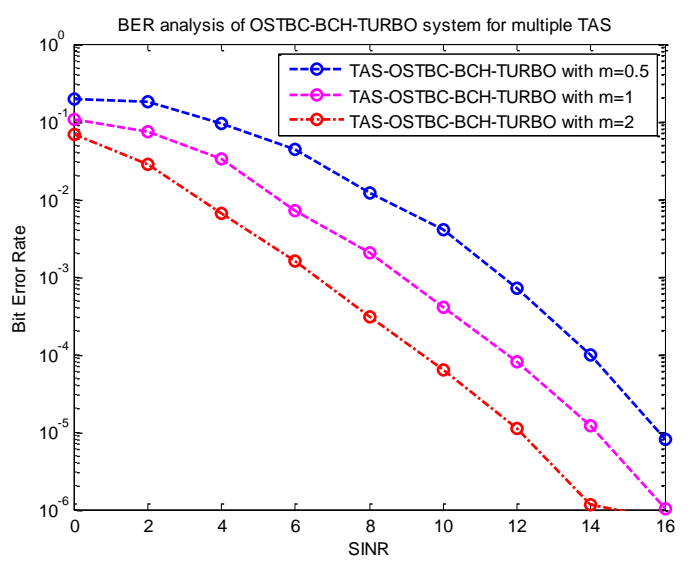

Figure 6 BER analysis of OSTBC-BCH-TURBO system for multiple TAS with $\mathrm{m}=0.5,1,2$.

\section{CONCLUSION}

The TAS-OSTBC system is proposed in this paper with new concatenation of BCH-TURBO codes in order to achieve the coding gain along with all the benefits of capacity and high error correction. The channel is assumed to be faded by Nakagami- $m$ fading channel coefficients. The performance of TAS-OSTBC system is first verified by concatenating with different FEC codes. It is observed that OSTBC-TURBO gives BER which is around 10-5 for SINR of $10 \mathrm{~dB}$ whereas the same will be obtained above $16 \mathrm{~dB}$ for TAS without FEC. Therefore the inner concatenation is carried out by using TURBO codes. Further, to improve the coding gain the outer concatenation is carried out by using $\mathrm{BCH}$ code. It is observed that the proposed system gives improvement of $5 \mathrm{~dB}$ at 10-6 as compared to concatenation of TURBO code only. The proposed system gives improved results for Nakagami- $\mathrm{m}$ fading with $\mathrm{m}=0.5$. The new concatenation of BCH-TURBO code to TAS-OSTBC system achieves optimum BER with coding gain.

\section{REFERENCES}

1. Andrew Molisch and M .Win, "MIMO systems with antenna selection," IEEE Microwave Mag., vol. 5, no. 2, pp. 46-56, March 2004

2. S. Prakash and I. McLoughlin, "Effects of channel prediction for transmit antenna selection with maximal ratio combining in Rayleigh fading", IEEE Transactions on Vehicular Technology., vol. 60, no. 6, pp. 2555-2568, 2011.

3. Z. Chen et al., "Analysis of transmit antenna selection/maximal-ratio combining in Rayleigh fading channels," IEEE Transactions on Vehicular Technology., vol. 54, no. 4, pp. 1312-1321, 2005.

4. Vaibhav Hendre et. al., "Performance analysis of transmit antenna selection with MRC in MIMO for image transmission in multipath fading channels", in International Journal of Electrical and Computer Engineering, Institute of Advance Engineering and Science (IAES), vol. 5, no.1, pp.119-128, 2015.

5. Vaibhav Hendre et. al., "Multiple transmit antenna selection in MIMO-OSTBC system in Nakagami- $\mathrm{m}$ fading channel", in Procedia Computer Science, Elsevier, vol. 54, pp. 558-565, 2015.

6. T. H. Liew, et.al., "Concatenated Space Time Block Codes And TCM, Turbo TCM, Convolutional as well as Turbo Codes,", IEEE Global Telecommunications Conference, vol. 3, pp. 1829-1833, 2000.

7. E. Ghayoula, et. al., "Improving MIMO System Performances By Concatenated LDPC Decoder To The MRC And STBC Receivers," World Symposium On Computer Networks And Information Security, pp. 102-108, 2015.

8. Haifen Yang, Guangjun Li, Wenfeng Lin and Yong Xiong, "Combining space time turbo trellis codes with transmit antenna selection", International Conference on Communications, Circuits and Systems, vol. 1 pp. 275-278, 2005.

9. A. Falou, et. al., "Transmit antenna selection for coded multiple-input dual-output systems", International Symposium on Turbo Codes and Iterative Information Processing (ISTC), Bremen, pp. 269-273, 2014. 
10. Sofi Naima et.al.. "Performance Improvement of MIMO-OSTBC System with BCH-TURBO Code In Rayleigh Fading Channel", Indonesian Journal of Electrical Engineering and Computer Science Vol. 11, No. 3, pp. 898 907, September 2018.

11. R. Agarwal et. al.. "SC-FDM-IDMA Scheme Employing BCH Coding," International Journal of Electrical and Computer Engineering (IJECE), vol. 7, no. 2, pp. 992-998, 2017.

12. Y. Oscar, et. al. , "On the frame error rate of Concatenated Turbo Codes," IEEE Transactions on communication, vol. 49, no. 4, APRIL 2001

13. G. Forney, "Concatenated Codes," Massachusetts Institute Of Technology Research Laboratory of Electronics, Technical Report 440, 3058assan3058r I, 1965.

14. Chen Z., et. al., "Error Performance of Maximal-Ratio Combining with Transmit Antenna Selection in Flat Nakagami- m Fading Channels," IEEE Transactions on Wireless Communications, vol. 8, no. 1, pp. 1312-1321, 2009.

15. S. Meraji, "Performance analysis of transmit antenna selection in Nakagami- $m$ fading channels," Wireless Personal Communication., vol. 43, pp. 327-333, Oct. 2007.

\section{AUTHORS PROFILE}

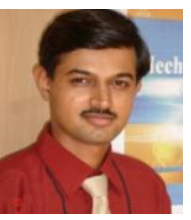

Dr. Vaibhav S. Hendre received his B.E (Electronics and Telecommunication Engineering) from University of Pune in 1999 and M. Tech. in Electroncis and Telecommunication from Dr. Babasaheb Ambedkar Technological University, Lonere in 2006. He received his Ph.D. from Sathyabama University, Chennai-600119, Tamil Nadu, India. He is currently serving as Professor and Head of Department of Electronics \& Telecommunication Engineering, G. H. Raisoni College of Engineering \& Management, Savitribai Phule Pune University, Pune-411048, Maharashtra, India. Having 19 years of academic experience, his fields of interest are Error Control coding, MIMO systems in Wireless Digital Communications and Cognitive Communication. He has published more than 12 papers in SCI and Scopus index Journal and 15 papers in various international conferences. He is a member of IEEE Communication Society and Life Member of ISTE.personal profile which contains their education details, their publications, research work, membership, achievements, with photo that will be maximum 200-400 words.

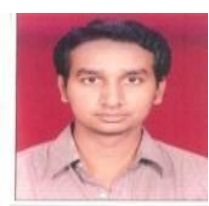

Nitin T. Deotale received his M. Tech. from Dr. Babasaheb Ambedkar Technological University, Lonere in 2006. He is a registered Ph.D Research Scholar of RTM Nagpur University, Nagpur, Maharashtra, India. He is currently serving as Assistant Professor in Department of Electronics \& Telecommunication Engineering, Lokmanya Tilak College of Engineering, Navi Mumbai-400709, Maharashtra, India. Having 10 years of academic experience, his fields of interest is Wireless Digital Communicationpersonal profile which contains their education details, their publications, research work, membership, achievements, with photo that will be maximum 200-400 words. 